\title{
EVALUATION OF THE QUANTUM AFFINE PRV DETERMINANTS
}

\author{
Anthony Joseph And Gail Letzter
}

\section{Introduction}

1.1. Let $U_{q}(\mathfrak{g})$ be the quantized enveloping algebra of the affine Lie algebra $\mathfrak{g}$. There is a well developed primitive ideal theory for the quantized enveloping algebra when the underlying Lie algebra is semisimple (see for example [J2].) The fundamental Verma module annihilator theorem is proved by comparing the factorization of the PRV determinant and the Shapavalov determinant. Recently, the first author and Todoric [JT] have shown how to define the more general KPRV determinant in the quantum affine case. The main result of this paper is a factorization of this determinant for the Borel case (see the terminology of $[\mathrm{JT}])$; henceforth, we refer to this object as the quantum affine PRV determinant.

Our paper is part of a program begun in [J3] by the first author and developed further in $[\mathrm{JT}]$, to extend the structure theory of $U_{q}(\mathfrak{g})$ for $\mathfrak{g}$ semisimple to the quantum affine case. This approach uses the regular completion $R(\hat{U})$ of the quantized enveloping algebra $U_{q}(\mathfrak{g})$ introduced in [J3]. By construction, the subquotients of $R(\hat{U})$ are the integrable modules which have a zero weight space with respect to the adjoint action. Thus $R(\hat{U})$ is a natural extension of the integral part of $U_{q}(\mathfrak{g})$. Furthermore, it has an appropriately large centre $Z(R(\hat{U}))$ which allows one to separate highest weight modules with highest weights lying in different Weyl group orbits. It has been shown [JT, 3.3] that the isotypical component $R(\hat{U})_{V}$, defined as the direct sum of simple submodules of an isomorphism class of a simple integrable admissible module $V$, is free over $Z(R(\hat{U}))$. Moreover the multiplicity of $V$ in the corresponding harmonic subspace $\mathbb{H}_{V}$ is exactly the dimension of the zero weight space $V_{0}$ of $V$. Fixing a particular basis of $V_{0}$, it is natural to form the matrix where the columns are images of this basis under linearly independent embeddings of $V$ into $\mathbb{H}_{V}$. Applying the Harish-Chandra projection and taking the determinant yields the PRV determinant $\mathcal{P}_{V}$ introduced in [JT, Definition 3.6]. Though we will not study it here, the construction in $[\mathrm{JT}]$ is defined in the more general parabolic case associating a determinant $\mathcal{P}_{\pi^{\prime}, V}$ to the pair $\left(\pi^{\prime}, V\right)$ where $\pi^{\prime}$ is a subset of the simple

\footnotetext{
Received January 15, 2001.

This work was partially supported by grant no. 7773 from the United States-Israel Binational Science Foundation (BSF), Jerusalem, Israel, and by the EC TMR network "Algebraic Lie Representations" grant no. ERB FMRX-CT97-0100. The second author was partially supported by NSA grant no. MDA 904-99-1-0033.
} 
roots $\pi$. We also remark that it is necessary to extend the base field $k(q)$ to the Laurent field $K:=k(q)\left(\left(\tau(\delta)^{2}\right)\right)$ where $\tau(\delta)$ is the torus element associated with the positive indivisible imaginary root $\delta$. Thus $\mathbb{H}_{V}$ is a $K$ vector space and $\mathcal{P}_{V}$ is defined up to an element of $K$.

1.2. We compute $\mathcal{P}_{V}$ in Theorem 4.7 by expressing it as the product of simple factors which, roughly speaking, correspond to linear terms upon specialization at $q=1$. Although our formula for $\mathcal{P}_{V}$ very closely resembles the result obtained in the semisimple case [JL, 3.6; J2, 8.2.10], there are several important distinctions which we note.

(i) Infinite Products. In contrast to the semisimple case, the $\mathcal{P}_{V}$ are infinite products. This means that they cannot be computed as in the semisimple case by examining their zeros.

(ii) The Underlying Simple Module V. In the semisimple case, the underlying simple module $V$ is finite-dimensional and hence is a highest weight module. However, in the affine case $V$ is neither a highest nor a lowest weight module (with the one exception when $V$ is the trivial module). Indeed $\tau(\delta)$ acts by 1 on $V$, so $V$ is of level 0 . Such modules are related to the more familiar finite dimensional modules obtained as quotients by forgetting the action of another central torus element, $\tau(\rho)$, associated to $\rho$, the sum of a choice of fundamental weights (notation 2.2). The exact relationship between these two categories of modules is unknown. However a correspondence can be anticipated from the somewhat analogous theory for $U(\mathfrak{g})$ modules (in the affine case) in which both classes of modules have been parametrized [CP, J4, G]. In any case, we need to know little of $V$ except that it is integrable and admissible, from which it follows that $V$ has a formal character invariant under the Weyl group. We remark that the action of $\tau(\rho)$ can not be forgotten and so $V$ will not be finite dimensional.

(iii) Disappearance of Imaginary Roots. We determine $\mathcal{P}_{V}$ up to a non-zero scalar in $K$. It is not a surprise therefore that only real roots occur in its factors. However, there is a "canonical" choice for the entries of the PRV determinants (see [JT, 3.6]) and in this choice the imaginary factors appear. These factors appear to be rather complicated and have not been worked out even in the simplest cases. The only result in this direction is a computation of their limit at $q=1$ in two special cases [J5, GJ]. In both examples, the $q=1$ imaginary factor takes a rather simple form.

(iv) The Quantum Ingredient. For $\mathfrak{g}$ semisimple, the PRV determinants were defined and computed first for the ordinary enveloping algebra $U(\mathfrak{g})$. Much of the argument was then modified to apply to the quantum semisimple case. Such inspiration from the classical set up is simply not available here. Indeed, we do not yet know how to define the PRV determinants for the classical enveloping algebra $U(\mathfrak{g})$ when $\mathfrak{g}$ is affine.

These distinctions, especially (i), made us very pessimistic that the methods used when $\mathfrak{g}$ is semisimple could be extended to the quantum affine case. In this paper, we use an approach which differs from the one developed in [J1] and later used to compute the quantum semisimple PRV determinant [J2, 8.2]. Our 
argument adapts the original proof of the factorization of the classical semisimple PRV determinant ([PRV, Theorem 4.2]). For each simple root $\alpha$, using the adjoint action of the corresponding copy of $U_{q}\left(\mathfrak{s l}_{2}\right)$, we write the determinant as a product of a Laurent polynomial in the torus element $\tau(\alpha)$ and a term invariant under the translated action of the reflection associated to $\alpha$. This information, combined with the translated action of the entire Weyl group and a description of the highest degree term, allow us to obtain the formula (Theorem 4.7) for $\mathcal{P}_{V}$.

1.3. In the classical case, the completion of $U(\mathfrak{g})$ used to recover the Casimir invariant (implicit in $[\mathrm{K}, 2.5]$ ) acts naturally on the $\mathcal{O}$ category. However, the quantum completion, in contrast to the classical one, also admits infinite sums of torus elements. Hence, it is not clear that $R(\hat{U})$ has a well-defined action on the $\mathcal{O}$ category. We would like to know that on replacing torus elements by appropriate powers of $q$, the corresponding infinite sums converge in the Krull topology. This is obviously a delicate question; indeed, the convergence already depends on the choice of the highest weight. In [J3, Sect. 6] we suggested how to show that $R(\hat{U})$ acts naturally on the subcategory $\mathcal{O}_{\mathbb{P}}$ of modules in $\mathcal{O}$ with simple factors whose highest weights lie in Weyl group translates of dominant weights. This approach works [J3, 6.2] for the "natural" generators of $Z(R(\hat{U}))$ over $K$.

In Section 5, we use our calculation of $\mathcal{P}_{V}$ to show that when $\operatorname{dim} V_{0}=1$, the corresponding harmonic part $\mathbb{H}_{V}$ can be taken so as to have a well-defined action on $\mathcal{O}_{\mathbb{P}}$. Assuming the dimension of $V_{0}$ is 1 is rather restrictive, but there are some non-trivial examples with this condition. Moreover, an immediate consequence of this result is that the subalgebra over $k(q)$ generated by $\mathbb{H}_{V}$ (which is much larger than the finite-dimensional vector space $\mathbb{H}_{V}$ ) has a well-defined action on $\mathcal{O}_{\mathbb{P}}$. This is rather encouraging, especially as a more direct approach appeared to fail (see [J5, 3.2]). The reason for this failure can be traced to a possible bad action of $K$. The elements of $K$ are central and so must act by a scalar on a highest weight module; however, it is clear that certain elements of $K$ will not have a well-defined action on $\mathcal{O}_{\mathbb{P}}$. Thus one is forced into making astute choices of bases.

\section{Notation and Preliminary Lemmas}

2.1. We shall follow the notation of [JT, Section 2] and briefly review the necessary definitions. The reader is also referred to [J2] and [J3] for undefined notions. The base field $k$ is assumed of characteristic zero and $q$ is an indeterminate.

2.2. Let $\mathfrak{g}$ be an affine Lie algebra. To avoid trivial technicalities we shall assume the root system indecomposable though the arguments go through for any affine or semisimple $\mathfrak{g}$ without significant changes. Let $\mathfrak{h}$ be a Cartan subalgebra, $\Delta$ (resp. $\Delta_{r e}$ ) the corresponding set of non-zero (resp. real) roots. Let $\pi$ be a choice of simple roots which is paired with the set $\left\{\alpha^{\vee}\right\}_{\alpha \in \pi}$ of coroots contained in $\mathfrak{h}$. Set $\Delta^{+}=\Delta \cap \mathbb{N} \pi, \Delta_{r e}^{+}=\Delta^{+} \cap \Delta_{r e}$. Recall that there is a unique positive imaginary root $\delta \in \mathbb{N} \pi$ indivisible in the sense that if $r \delta \in \mathbb{N} \pi: r \in \mathbb{Q}$ 
then $r \in \mathbb{Z}$. Let $P(\pi)$ denote the set of $\mathbb{Z}$ linear combinations of a choice of fundamental weights and of $\delta$ and let $P^{+}(\pi)$ denote the set of dominant integral weights. Write $($,$) for the non-degenerate symmetric bilinear form defined in$ [K, Chapter 2], normalized so that $(\lambda, \mu)$ is an integer for all $\lambda, \mu$ in $P(\pi)$. Let $\rho$ be the sum of the fundamental weights. The integral torus $T$ is the corresponding multiplicative group and we let $\tau: P(\pi) \rightarrow T$ be the corresponding isomorphism (satisfying $\tau(\lambda+\mu)=\tau(\lambda) \tau(\mu)$, for all $\lambda, \mu \in P(\pi)$ ). Set $t_{\alpha}=\tau(\alpha)$, for all $\alpha \in \mathbb{Z} \pi$.

2.3. By definition the (simply-connected) Drinfeld-Jimbo quantized enveloping algebra $U_{q}(\mathfrak{g}$ ) (or simply, $U$ ) is the Hopf algebra generated over the subgroup $T$ by weight vectors $e_{\alpha}, f_{-\alpha}: \alpha \in \pi$ satisfying the relations described specifically in $[\mathrm{J} 2,5.1 .1]$. Given $a$ and $b$ in $U$, we write $(a d a) b$ to denote the quantum adjoint action of $a$ on $b$ (see for example [JT 2.4] or [J4, 1.3.1 and 5.3.1].) In particular, we have that

$$
\left(a d e_{\alpha}\right) a=e_{\alpha} a t_{\alpha}-a e_{\alpha} t_{\alpha} \quad \text { and } \quad\left(a d f_{-\alpha}\right) a=f_{-\alpha} a-t_{\alpha} a t_{\alpha}^{-1} f_{-\alpha}
$$

for all $a \in U$.

2.4. Recall $[\mathrm{J} 2,7.1 .17]$ the translated action of the Weyl group $W$ on $T$ given by

$$
w \cdot \tau(\beta)=\tau(w \beta) q^{(\rho, w \beta-\beta)}
$$

for all $w \in W$ and $\beta \in \mathbb{Z} \pi$. It is convenient to define $r_{\beta}:=\tau(\beta) q^{(\rho, \beta)}$, for all $\beta \in \mathbb{Z} \pi$. In terms of these new coordinates, one has $s_{\beta} \cdot r_{\gamma}=r_{s_{\beta} \gamma}$, for all $\beta \in \Delta_{r e}$ and $\gamma \in \mathbb{Z} \pi$. Set $A_{\pi}=k(q)\left[\left[r_{\alpha}^{2}: \alpha \in \pi\right]\right]$.

Lemma. $A_{\pi}^{W}$ reduces to scalars (in $K$ ).

Proof. The assertion is equivalent to every $W$ orbit in $\mathbb{N} \pi$ being a singleton. Although this assertion is presumably known, we give a proof for the sake of completeness.

Recall that an affine Lie algebra $\mathfrak{g}$ can be built from a simple Lie algebra $\mathfrak{g}_{0}$ with Cartan subalgebra $\mathfrak{h}_{0}$ and Weyl group $W_{0}$. Let $\pi_{0}$ be a choice of simple roots for $\left(\mathfrak{g}_{0}, \mathfrak{h}_{0}\right)$ and let $\varphi$ denote the corresponding highest root. Then $\pi=$ $\left\{\pi_{0}, \alpha_{0}\right\}$ where $\alpha_{0}=-\varphi+\delta$. For each $\beta \in \Delta_{r e}$ set $\beta^{\vee}=2 \beta /(\beta, \beta)$ and let $s_{\beta}$ denote the corresponding reflection. In particular setting $s_{0}=s_{\alpha_{0}}$ one has $s_{0} \lambda=\lambda-\left(\alpha_{0}^{\vee}, \lambda\right)(-\varphi+\delta)$ for all $\lambda \in P(\pi)$. Consequently for all $\lambda \in \mathbb{Z} \pi$ one has $s_{0} \lambda=\lambda+\left(\varphi^{\vee}, \lambda\right)(-\varphi+\delta)=s_{\varphi} \lambda+\left(\varphi^{\vee}, \lambda\right) \delta$ and so $s_{\varphi} s_{0} \lambda=\lambda+\left(\varphi^{\vee}, \lambda\right) \delta$.

Consider a $W$ orbit in $\mathbb{Z} \pi$. By the above, a typical element takes the form $\beta+r \delta$, for some $\beta \in \mathbb{Z} \pi_{0}$ and $r \in \mathbb{Z}$. If $\beta=0$, this orbit is a singleton. Otherwise we can assume $\beta$ to be non-zero and $W_{0}$ dominant. Then $(\varphi, \beta) \neq 0$. By our previous formula $\left(s_{\varphi} s_{0}\right)^{n} \beta=\beta+n\left(\varphi^{\vee}, \beta\right) \delta$, for all $n \in \mathbb{Z}$ and so the above orbit cannot lie in $\mathbb{N} \pi$. 
2.5. Given a subset $\pi^{\prime}$ of $\pi$, let $G_{\pi^{\prime}}^{+}$(resp. $U_{\pi^{\prime}}^{-}$) denote the subalgebra of $U$ generated by $t_{\alpha} e_{\alpha}$ (resp. $f_{-\alpha}$ ) for $\alpha \in \pi^{\prime}$, and let $U_{\pi^{\prime}}$ be the subalgebra generated by the torus $T, G_{\pi^{\prime}}^{+}$, and $U_{\pi^{\prime}}^{-}$. Recall [JT, 5.1] that there is a triangular decomposition (due to M. Kébé $[\mathrm{Ke}]$ )

$$
S_{\pi^{\prime}}^{-} \otimes U_{\pi^{\prime}} \otimes S_{\pi^{\prime}}^{+} \stackrel{\sim}{\longrightarrow} U_{q}(\mathfrak{g})
$$

where $S_{\pi^{\prime}}^{-}\left(\right.$resp. $\left.S_{\pi^{\prime}}^{+}\right)$is the subalgebra of $U$ generated by $\left(\right.$ ad $\left.G_{\pi^{\prime}}^{+}\right) G_{\pi \backslash \pi^{\prime}}^{+}$(resp. $\left(\right.$ ad $\left.U_{\pi^{\prime}}^{-}\right) U_{\pi \backslash \pi^{\prime}}^{-}$). It defines (as usual using the augmentations of $S_{\pi^{\prime}}^{ \pm}$) a projection $\mathcal{P}_{\pi^{\prime}}$ of $U_{q}(\mathfrak{g})$ onto $U_{\pi^{\prime}}$. When $\pi^{\prime}$ is the empty set, $U_{\pi^{\prime}}=k(q) T$ and $\mathcal{P}_{\pi^{\prime}}$ is just the usual Harish-Chandra projection. We denote it by $\mathcal{P}$. When $\pi^{\prime}=\{\alpha\}, U_{\pi^{\prime}}$ is the Hopf subalgebra $U_{q}\left(\mathfrak{r}_{\alpha}\right)$ of $U_{q}(\mathfrak{g})$ generated by $e_{\alpha}, f_{-\alpha}$ over $T$. In this case we denote $\mathcal{P}_{\pi^{\prime}}$ by $\mathcal{P}_{\alpha}$ and $U_{\pi^{\prime}}$ by $U_{\alpha}$. Clearly $\mathcal{P} \mathcal{P}_{\alpha}=\mathcal{P}$. One checks using the adjoint action (see Section 2.3) as in [JT, 5.1] that both $S_{\pi^{\prime}}^{+}$and $S_{\pi^{\prime}}^{-}$are $\left(\right.$ad $\left.U_{\pi^{\prime}}\right)$ stable. It follows that $\mathcal{P}_{\pi^{\prime}}$ commutes with the adjoint action of $U_{\pi^{\prime}}$.

2.6. Let $I(U)$ denote the integral part of $U$ (see [J2, 7.1]). In particular, $I(U)$ is the set of all elements $a$ in $U$ which generate a finite-dimensional (ad $\left.U_{\alpha}\right)$ module for each $\alpha \in \pi$. By say [J2, 1.3.1], it is a subalgebra of $U$. When $\mathfrak{g}$ is semisimple, $I(U)$ is just the locally finite part $F(U)$ of $U$ and admits an isomorphism

$$
Z(U) \otimes \mathbb{H} \stackrel{\sim}{\longrightarrow} F(U)
$$

of vector spaces via the multiplication map where $Z(U)$ is the center of $U$ and $\mathbb{H}$ is an ad $U$ module referred to as the harmonics (see [J2, 7.3]).

Recently, the first author and Todoric ([JT]) obtained a restricted analog of this isomorphism in the affine case by passing to the regular completion $R(\hat{U})$ of $U$. We use the modified version of $R(\hat{U})$ defined in [JT, 3.4]. In particular, recall that $K$ is the Laurent field $k(q)\left(\left(\tau(\delta)^{2}\right)\right)$. Then $R(\hat{U})$ is the $K$ algebra generated by the version of the regular completion, also denoted by $R(\hat{U})$, introduced in [J3, Section 4]. Let $V$ be a simple integrable admissible module and set $R(\hat{U})_{V}$ equal to the isotypical component of the socle of $R(\hat{U})$ of type $V$. Then [JT, Theorem 3.4] there exists an $(a d U)$ submodule $\mathbb{H}_{V}$ of $R(\hat{U})_{V}$ such that the multiplication map gives a linear isomorphism

$$
Z(R(\hat{U})) \otimes \mathbb{H}_{V} \stackrel{\sim}{\longrightarrow} R(\hat{U})_{V}
$$

where $Z(R(\hat{U}))$ is the center of $R(\hat{U})$. Although the choice of $\mathbb{H}_{V}$ is not unique, any choice is a direct sum of $\operatorname{dim} V_{0}$ copies of $V$ ([JT, 3.1]).

The regular completion $R(\hat{U})$ of $U$ consists of certain infinite sums of elements of $I(U)$ over $K$. Hence the Harish-Chandra projection $\mathcal{P}$ extends to $R(\hat{U})$ and its image lies in $\hat{U}_{0}:=k(q) T\left[\left[r_{\alpha}^{2}: \alpha \in \pi\right]\right]$. Let $\left\{v_{i}\right\}$ be a basis for the zero weight space $V_{0}$ of $V$, and $\left\{\varphi_{j}\right\}$ be a basis for $\operatorname{Hom}\left(V, \mathbb{H}_{V}\right)$. Set $v_{i, j}=\varphi_{j}\left(v_{i}\right)$. Then the PRV determinant associated to $V$ is

$$
\mathcal{P}_{V}=\operatorname{det} \mathcal{P}\left(v_{i, j}\right) \text {. }
$$

It is determined up to a nonzero element of $K$. 
2.7. The map $\mathcal{P}_{\alpha}$ can also be extended to $R(\hat{U})$ and its image lies in the regular completion $R\left(\hat{U}_{\alpha}\right)$ of $U_{\alpha}$. Recall that $U_{\alpha}$ is the quantized enveloping algebra of the Lie algebra $\mathfrak{r}_{\alpha}$ (see Section 2.5). Note that $\operatorname{dim} \mathfrak{r}_{\alpha}<\infty$, but $\mathfrak{r}_{\alpha}$ is not semisimple. Hence the appropriate definition of $R\left(\hat{U}_{\alpha}\right)$ is $I\left(U_{\alpha}\right) U^{\alpha}$, where $U^{\alpha}:=\left\{u \in \hat{U}^{0} \mid s_{\alpha} \cdot u=u\right\}$. By [J2, 7.3.8(ii)], $I\left(U_{\alpha}\right)$ is a free module over its centre $Z_{\alpha}$. More precisely we have a $Z_{\alpha}$ module isomorphism via the multiplication map

$$
I\left(U_{\alpha}\right) \stackrel{\sim}{\longleftarrow} \mathbb{H}_{\alpha} \otimes Z_{\alpha}
$$

with "harmonic part" $\mathbb{H}_{\alpha}$ being an ad $U_{\alpha}$ submodule. This does not determine $\mathbb{H}_{\alpha}$ uniquely. However, one choice for $\mathbb{H}_{\alpha}$ is the sum of the (ad $U_{\alpha}$ ) modules generated by the $e_{\alpha}^{n}, n \geq 0$. Since $e_{\alpha}^{n}$ generates a $2 n+1$-dimensional simple module, we may write

$$
\mathbb{H}_{\alpha}=\bigoplus_{n \in \mathbb{N}} V^{(n)}
$$

with $V^{(n)}$ a (unique up to isomorphism) simple $U_{\alpha}$ module of dimension $2 n+1$. Let $v^{(n)}$ be a non-zero element of the one dimensional zero weight space $V_{0}^{(n)}$ of $V^{(n)}$. Note that $\mathcal{P}\left(v^{(n)}\right)$ corresponds to the PRV determinant of $V^{(n)}$ associated to the copy of the quantized enveloping algebra $U_{q}\left(\mathfrak{s l}_{2}\right)$ generated by $e_{\alpha}, f_{-\alpha}$, and $t_{\alpha}^{ \pm 1}$. Thus by $[\mathrm{J} 2,8.2 .10]$ we have

$$
\mathcal{P}\left(v^{(n)}\right)=\prod_{m=1}^{n}\left(r_{\alpha}-q^{m(\alpha, \alpha)} r_{\alpha}^{-1}\right),
$$

up to a non-zero scalar. We write the product in the right hand side of $(*)$ simply as $S(n)$.

Let $T^{\alpha}$ denote the subgroup of $T$ generated by the elements $\tau(\beta): \beta \in \mathbb{Z} \pi$ satisfying $(\beta, \alpha)=0$. By $[\mathrm{J} 2,4.3 .4]$ it follows that $Z_{\alpha}$ is the polynomial ring over $k(q) T^{\alpha}$ generated by an element $z_{\alpha}$. Moreover

$$
\mathcal{P}\left(z_{\alpha}\right)=\left(r_{\alpha}+r_{\alpha}^{-1}\right) \text {. }
$$

Clearly $T^{\alpha} \subset\left(\hat{U}^{0}\right)^{s_{\alpha}}$. which also contains $\mathcal{P}\left(z_{\alpha}\right)$. From the above we deduce the following

Lemma. Let $V^{\prime}$ be a simple ad $U_{\alpha}$ submodule of $R\left(\hat{U}_{\alpha}\right)$ of dimension $2 n+1$. Then $\mathcal{P}\left(V^{\prime}\right) \subset S(n) U^{\alpha}$.

\section{Distributivity of the Baumann Filtration}

3.1. Fix a simple integrable admissible module $V$ with a non-zero (and hence finite dimensional) zero weight space $V_{0}$. One of the important steps in calculating the quantum semisimple PRV determinant is the computation of its highest degree term with respect to the filtration on $U$ defined in $[\mathrm{J} 3,5.3 .1]$ (see also $[\mathrm{JT}, 3.2])$. In order to compute this term in the affine case, we transfer the distributivity property of this filtration on $U$ to the Baumann filtration $\mathcal{F}$ on subspaces of $V_{0}$. 
3.2. Let $P_{0}^{+}(\pi)$ be the additive semigroup generated by the (choice of) fundamental weights. Define an ordering $\preccurlyeq$ on $P_{0}^{+}(\pi)$ through $\mu \preccurlyeq \nu$ given by $\nu-\mu \in P_{0}^{+}(\pi)$. Let $M$ be a subspace of $V_{0}$. For each $\mu \in P_{0}^{+}(\pi)$, set

$$
\mathcal{F}^{\mu}(M)=\left\{v \in M \mid e_{\alpha}^{\alpha^{\vee}(\mu)+1} v=0, \forall \alpha \in \pi\right\},
$$

and

$$
\mathcal{F}_{\mu}(M)=\mathcal{F}^{\mu}(M) / \sum_{\nu \prec \mu} \mathcal{F}^{\nu}(M)
$$

Given two elements $\nu, \mu$ of $P_{0}^{+}(\pi)$, let $\nu \cap \mu$ be the unique maximal element in $P_{0}^{+}(\pi)$ which satisfies $(\nu \cap \mu) \preccurlyeq \min (\nu, \mu)([\mathrm{J} 2,7.3])$. The definition of $\mathcal{F}$ implies that $\mathcal{F}^{\mu}(M) \cap \mathcal{F}^{\nu}(M)=\mathcal{F}^{\mu \cap \nu}(M)$ for all $\mu, \nu \in P_{0}^{+}(\pi)$. The filtration $\mathcal{F}$ on $M$ is said to be distributive if

$$
\mathcal{F}^{\mu}(M) \cap\left(\sum_{j=1}^{n} \mathcal{F}^{\nu_{j}}(M)\right)=\sum_{j=1}^{n}\left(\mathcal{F}^{\mu \cap \nu_{j}}(M)\right)
$$

for all $\mu, \nu_{1}, \ldots, \nu_{n}$ in $P_{0}^{+}(\pi)$.

Lemma. Let $M$ be a subspace of $V_{0}$ and $\mu \in P_{0}^{+}(\pi)$. If $\mathcal{F}^{\mu}(M)$ is nonzero, then there exists $\mu^{\prime} \in P_{0}^{+}(\pi)$ such that $\mu^{\prime} \preccurlyeq \mu$ and $\mathcal{F}_{\mu^{\prime}}(M)$ is nonzero.

Proof. Let $\mu^{\prime}$ be a minimal element of $P_{0}^{+}(\pi)$ with respect to the properties that $\mu^{\prime} \preccurlyeq \mu$ and $\mathcal{F}^{\mu^{\prime}}(M)$ is nonzero. Suppose $\nu \prec \mu$. By choice of $\mu^{\prime}, \mathcal{F}^{\nu}(M)$ is zero. Hence $\sum_{\nu \prec \mu^{\prime}} \mathcal{F}^{\nu}(M)=0$ and $\mathcal{F}_{\mu^{\prime}}(M)$ is isomorphic to $\mathcal{F}^{\mu^{\prime}}(M)$ as vector spaces. In particular, $\mathcal{F}_{\mu^{\prime}}(M) \neq 0$.

3.3. Given a subspace $M$ of $V_{0}$, define $\operatorname{gr}_{\mathcal{F}} M$ to be the vector space

$$
\operatorname{gr}_{\mathcal{F}} M=\bigoplus_{\mu} \mathcal{F}_{\mu}(M)
$$

Since $M$ is finite dimensional, $\mathcal{F}_{\mu}(V)$ is the zero vector space when $0 \prec \prec \mu$. Thus we can choose a finite subset $\left\{\mu_{1}, \ldots, \mu_{n}\right\}$ of $P_{0}^{+}(\pi)$ such that

$$
\operatorname{gr}_{\mathcal{F}} M=\bigoplus_{j=1}^{n} \mathcal{F}_{\mu_{j}}(M)
$$

Lemma. If $\mathcal{F}$ is distributive on $M$, then $\operatorname{dim} \operatorname{gr}_{\mathcal{F}} M=\operatorname{dim} M$.

Proof. Reorder the set $\left\{\mu_{1}, \ldots, \mu_{n}\right\}$ so that $\mu_{i} \prec \mu_{j}$ implies $i<j$. For each $i$, choose a subset $B\left(\mu_{i}\right)$ of $\mathcal{F}^{\mu_{i}}(M)$ such that $\left\{\mathrm{gr} v \mid v \in B\left(\mu_{i}\right)\right\}$ is a basis for $\mathcal{F}_{\mu_{i}}(M)$. We argue that that the union $B$ of the $B\left(\mu_{i}\right)$ is a basis for $M$. First, using distributivity and induction on $n$, we show that $B$ is a linearly independent subset of $M$.

By the ordering of the set $\left\{\mu_{1}, \ldots, \mu_{n}\right\}$ and Lemma $3.2, \mathcal{F}^{\nu}(M)=0$ for all $\nu \prec \mu_{1}$. Thus $\mathcal{F}^{\mu_{1}}(M) \cong \mathcal{F}_{\mu_{1}}(M)$ as vector spaces. It follows that $B\left(\mu_{1}\right)$ is a linearly independent subset of $M$. 
Now assume that $B_{k}=B\left(\mu_{1}\right) \cup \cdots B\left(\mu_{k}\right)$ is a linearly independent subset of $M$. Let $b \in \operatorname{Span} B\left(\mu_{k+1}\right)$. Suppose that $b \in \operatorname{Span} B_{k}$. Then by the distributive property,

$$
b \in \mathcal{F}^{\mu_{k+1}}(M) \cap\left(\sum_{i=1}^{k} \mathcal{F}^{\mu_{i}}(M)\right) \subseteq \sum_{i=1}^{k} \mathcal{F}^{\mu_{k+1} \cap \mu_{i}}(M) .
$$

Note that $\mu_{k+1} \cap \mu_{i} \prec \mu_{k+1}$ for all $1 \leq i \leq k$. Thus $b \in \sum_{\nu \prec \mu_{k+1}} \mathcal{F}^{\nu}(M)$ and the image of $b \in \mathcal{F}_{\mu_{k+1}}(M)$ is zero. This contradicts the fact that $b \in B\left(\mu_{k+1}\right)$ and the image of $B\left(\mu_{k+1}\right)$ in $\mathcal{F}_{\mu_{k+1}}(M)$ is a basis. Thus by induction, $B$ is a linearly independent subset of $M$.

Suppose that $B$ does not span $M$. Given $b \in M$, set $S_{b}=\left\{\mu \in P_{0}^{+}(\pi) \mid b \in\right.$ $\left.\mathcal{F}^{\mu}(M)\right\}$. Choose $\eta \in P_{0}^{+}(\pi)$ minimal with respect to the property that $\eta \in S_{b}$ for some $b \in M-\operatorname{Span}(B)$. Let $m$ be an element in $M$ such that $\eta \in S_{m}$ and $m \notin \operatorname{Span}(B)$. So $m \in \mathcal{F}^{\eta}(M)$. Now suppose that $m \in \sum_{\nu \prec \eta} \mathcal{F}^{\nu}(M)$. Hence $m=\sum m_{\nu}$ where each $m_{\nu} \in \mathcal{F}^{\nu}(M)$. But then by the choice of $\eta$, $m_{\nu} \in \operatorname{Span}(B)$ for each $\nu$. Thus $m \in \operatorname{Span}(B)$, a contradiction. Hence we may assume that $m \notin \sum_{\nu \prec \eta} \mathcal{F}^{\nu}(M)$. It follows that $\mathcal{F}_{\eta}(M)$ is nonzero and so $\eta=\mu_{i}$ for some $i$. Then there exists an element $w \in \operatorname{Span}\left(B\left(\mu_{i}\right)\right)$ such that $m-w \in$ $\sum_{\nu \prec \mu_{i}} \mathcal{F}^{\mu_{i}}(M)$. By the minimality of $\eta=\mu_{i}$, it follows that $m-w \in \operatorname{Span}(B)$ which forces $m \in \operatorname{Span}(B)$, a contradiction. Thus $\operatorname{Span}(B)=M$.

3.4. Given $\mu \in P^{+}(\pi)$, let $V(\mu)$ (resp. $V(-\mu)$ ) denote the simple highest weight module of highest weight $\mu$ with highest (resp. lowest) generating vector $v_{\mu}$ (resp. $\left.v_{-\mu}\right)$. It is straightforward to check that the vector $v_{\mu} \otimes v_{-\mu}$ generates $V(\mu) \otimes V(-\mu)$ as a $U$ module.

Given a left $U$ weight module $N$, let $N^{\#}$ be its graded dual as defined in $[\mathrm{J} 3,2.6]$ using the antipode. Note that the $U$ modules $V(\mu)^{\#}$ and $V(-\mu)$ are isomorphic and thus can be identified with each other.

Suppose that $\nu$ is another element of $P^{+}(\pi)$ and assume that $\nu \prec \mu$. By [J3, Remark 5.2], the annihilator of $v_{\mu} \otimes v_{-\mu}$ in $U$ is a subset of the annihilator of $v_{\nu} \otimes v_{-\nu}$ in $U$. This inclusion of annihilators induces a surjective $U$ module map $\psi_{\mu, \nu}$ from $V(\mu) \otimes V(-\mu)$ onto $V(\nu) \otimes V(-\nu)$ such that $\psi_{\mu, \nu}\left(v_{\mu} \otimes v_{-\mu}\right)=v_{\nu} \otimes v_{-\nu}$. The transpose yields an injective $U$ module map:

$$
\psi_{\mu, \nu}^{*}:(V(\nu) \otimes V(-\nu))^{\#} \rightarrow(V(\mu) \otimes V(-\mu))^{\#} .
$$

Let $\Psi_{\mu, \nu}^{*}$ be the map

$$
\Psi_{\mu, \nu}^{*}: \operatorname{Hom}\left(V^{\#},(V(\nu) \otimes V(-\nu))^{\#}\right) \rightarrow \operatorname{Hom}\left(V^{\#},(V(\mu) \otimes V(-\mu))^{\#}\right)
$$

induced by $\psi_{\mu, \nu}^{*}$.

3.5. For each $v \in \mathcal{F}^{\mu}\left(V_{0}\right)$, one may define [J3, 5.2] a $U$ module map $\varphi_{v}^{\mu} \in$ $\operatorname{Hom}(V(\mu) \otimes V(-\mu), V)$ by $\varphi_{v}^{\mu}\left(a\left(v_{\mu} \otimes v_{-\mu}\right)\right)=a v$ for all $a \in U$. The transpose $\varphi_{v}^{\mu, *}$ restricts to a $U$ module map from $V^{\#}$ to $(V(\mu) \otimes V(-\mu))^{\#}$ given by 
$\varphi_{v}^{\mu, *}(\xi)\left(a\left(v_{\mu} \otimes v_{-\mu}\right)\right)=\xi(a v)$ for all $a \in U$ and $\xi \in V(\mu) \otimes V(-\mu)$. Let $\Phi_{\mu}$ be the linear map

$$
\Phi_{\mu}: \mathcal{F}^{\mu}\left(V_{0}\right) \rightarrow \operatorname{Hom}\left(V^{\#},(V(\mu) \otimes V(-\mu))^{\#}\right)
$$

defined by $\Phi_{\mu}(v)=\varphi_{v}^{\mu, *}$. Let $T(\operatorname{End} V(\mu))$ denote the submodule of $\operatorname{End} V(\mu)$ spanned by weight vectors (with respect to the diagonal action). As noted in [JT, 3.1] Frobenius reciprocity gives an isomorphism $(V(\mu) \otimes V(-\mu))^{*} \stackrel{\sim}{\longrightarrow}$ $\operatorname{Hom}\left(V(\mu), V(\mu)^{\#, *}\right)$ and, hence, an embedding $(V(\mu) \otimes V(-\mu))^{\#} \hookrightarrow$

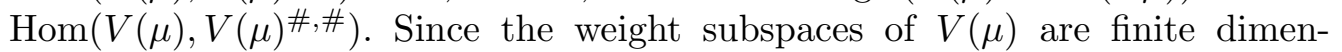
sional (it is here that we need $\tau(\rho)$ to act) one obtains an isomorphism $(V(\mu) \otimes$ $V(-\mu))^{\#} \stackrel{\sim}{\longrightarrow} T(\operatorname{End} V(\mu))$. Therefore, as in [JT, 3.1], the assertion in [J3, 5.12] ensures that $\Phi_{\mu}$ is a vector space isomorphism.

Let $i_{\mu, \nu}$ denote the inclusion map from $\mathcal{F}^{\nu}\left(V_{0}\right)$ into $\mathcal{F}^{\mu}\left(V_{0}\right)$.

Lemma. For all $\mu$ and $\nu$ in $P_{0}^{+}(\pi)$ with $\nu \prec \mu, \Psi_{\mu, \nu}^{*} \Phi_{\nu}=\Phi_{\mu} i_{\mu, \nu}$.

Proof. Since $v_{\mu} \otimes v_{-\mu}$ generates $V(\mu) \otimes V(-\mu)$ as a $U$ module, it is sufficient to check that $\left(\Psi_{\mu, \nu}^{*}\left(\Phi_{\nu}(v)\right)(\zeta)\right)\left(a\left(v_{\mu} \otimes v_{-\mu}\right)\right)=\left(\left(\Phi_{\mu} i_{\mu, \nu}(v)\right)(\zeta)\right)\left(a\left(v_{\mu} \otimes v_{-\mu}\right)\right)$ for all $v \in \mathcal{F}^{\nu}\left(V_{0}\right), a \in U$, and $\zeta \in V^{\#}$. We have

$$
\begin{aligned}
& \left(\Psi_{\mu, \nu}^{*}\left(\Phi_{\nu}(v)\right)(\zeta)\right)\left(a\left(v_{\mu} \otimes v_{-\mu}\right)\right)=\left(\left(\Psi_{\mu, \nu}^{*} \varphi_{v}^{\nu, *}\right)(\zeta)\right)\left(a\left(v_{\mu} \otimes v_{-\mu}\right)\right) \\
& \quad=\left(\varphi_{v}^{\nu, *}(\zeta)\right)\left(\psi_{\mu, \nu}\left(a\left(v_{\mu} \otimes v_{-\mu}\right)\right)\right)=\left(\varphi_{v}^{\nu, *}(\zeta)\right)\left(a\left(\psi_{\mu, \nu}\left(v_{\mu} \otimes v_{-\mu}\right)\right)\right) \\
& \quad=\left(\varphi_{v}^{\nu, *}(\zeta)\right)\left(a\left(v_{\nu} \otimes v_{-\nu}\right)\right)=\zeta(a v) .
\end{aligned}
$$

On the other hand

$$
\begin{aligned}
\left(\left(\Phi_{\mu}\left(i_{\mu, \nu}(v)\right)\right)(\zeta)\right)\left(a\left(v_{\mu} \otimes v_{-\mu}\right)\right) & =\left(\Phi_{\mu}(v)(\zeta)\right)\left(a\left(v_{\mu} \otimes v_{-\mu}\right)\right) \\
& =\varphi_{v}^{\mu, *}(\zeta)\left(a\left(v_{\mu} \otimes v_{-\mu}\right)\right)=\zeta(a v) .
\end{aligned}
$$

3.6. Let gr $U$ denote the associated graded algebra of $U$ with respect to the ad $U$ filtration on $U$ defined in [J2, 5.3.1]. Let $K(\mu)^{-}$be the vector subspace of $U^{-}$and let $K(-\mu)^{+}$be the vector subspace of $U^{+}$such that $($ad $U) \operatorname{gr} \tau(-2 \mu)=$ $K(\mu)^{-} \operatorname{gr} \tau(-2 \mu) K(-\mu)^{+}=: G(\mu)([\mathrm{J} 2,7.1 .1])$.

Let $\left\{v_{i}\right\}$ be a basis for $V(\mu)$ composed of weight vectors and $\left\{\xi_{i}\right\}$ the dual basis in $V(-\mu)=V(\mu)^{\#}$. With respect to the natural embedding $V(\mu) \otimes V(-\mu) \hookrightarrow$ $\operatorname{End} V(\mu)$, we can write each element of $\operatorname{End} V(\mu)$ uniquely as an infinite sum $\sum w_{i} \otimes \xi_{i}: w_{i} \in V(\mu)$. Identifying $V(\mu) \otimes V(-\mu)$ with $G(\mu)$ via [J2, 7.1.1], it follows that its completion $\hat{G}(\mu)$, as defined in [JT, 3.2], identifies with $\operatorname{End} V(\mu)$. Set

$$
G(U)=\bigoplus_{\mu \in P^{+}(\pi)} G(\mu) \text { and } \hat{G}(U)=\bigoplus_{\mu \in P^{+}(\pi)} \hat{G}(\mu) .
$$

As noted in [JT,3.2] the algebra structure on $G(U)$, detailed in [J2, Lemma 7.1.7], extends to $\hat{G}(U)$. We may suppose that $v_{-\mu}\left(v_{\mu}\right)=1$ and then $\operatorname{gr} \tau(-2 \mu)$ identifies with $v_{\mu} \otimes v_{-\mu}$. 
Let $y_{\mu}$ be a basis vector for the $(a d U)$ invariant subspace of $\hat{G}(\mu)$. By [J2, 7.3.3] and rescaling if necessary, we can write

$$
y_{\mu} \in \tau(-2 \mu)+\sum_{\beta>0} K(\mu)_{-\beta}^{-} \operatorname{gr} \tau(-2 \mu) K(-\mu)_{\beta}^{+}
$$

Note that this sum is infinite, but there is only a finite contribution from each space $K(\mu)_{-\beta}^{-} \operatorname{gr} \tau(-2 \mu) K(-\mu)_{\beta}^{+}$. Under the identification of $\operatorname{End} V(\mu)$ with $\hat{G}(\mu)$, $y_{\mu}$ becomes an element of $v_{\mu} \otimes v_{-\mu}+\sum_{\beta>0} V(\mu)_{\mu-\beta} \otimes V(-\mu)_{-\mu+\beta}$.

Let $\nu \in P^{+}(\pi)$ such that $\nu \prec \mu$. Since the $\hat{G}(\mu): \mu \in P^{+}(\pi)$ form a gradation of $\hat{G}(U)$, one has $y_{\mu-\nu} \hat{G}(\nu) \subset \hat{G}(\mu)$. Thus multiplication by $y_{\mu-\nu}$ induces a $U$ module map

$$
Y_{\nu, \mu}: \operatorname{End} V(\nu) \rightarrow \operatorname{End} V(\mu) .
$$

Moreover, [JT, Lemma 3.2] implies that this map is injective.

For each $f \in \operatorname{End} V(\nu)$, there is a scalar $b$ such that

$$
f \in b\left(v_{\nu} \otimes v_{-\nu}\right)+\sum_{\beta+\gamma>0} V(\nu)_{\nu-\gamma} \otimes V(-\nu)_{-\nu+\beta} .
$$

Note that $\tau(-2(\mu-\nu)) \tau(-2 \nu)=\tau(-2 \mu)$. Using the above identifications, it follows that the product $\left(v_{\nu} \otimes v_{-\nu}\right)\left(v_{\mu-\nu} \otimes v_{-\mu+\nu}\right)$ in $\hat{G}(U)$ equals $v_{\mu} \otimes v_{\mu}$. Therefore,

$$
Y_{\nu, \mu}(f) \in b\left(v_{\mu} \otimes v_{-\mu}\right)+\sum_{\beta+\gamma>0} V(\mu)_{\mu-\gamma} \otimes V(-\mu)_{-\mu+\beta} .
$$

Lemma. The restriction of $Y_{\nu, \mu}$ to $(V(\nu) \otimes V(-\nu))^{\#}$ equals $\psi_{\mu, \nu}^{*}$.

Proof. Consider $f \in(V(\nu) \otimes V(-\nu))^{\#}$ and write $f$ as in $(*)$. Then $\psi_{\nu, \mu}^{*}(f)\left(v_{\mu} \otimes\right.$ $\left.v_{-\mu}\right)=f\left(v_{\nu} \otimes v_{-\nu}\right)=b$. On the other hand, $(* *)$ implies that $Y_{\nu, \mu}(f)\left(v_{\mu} \otimes v_{-\mu}\right)=$ $\left(b\left(v_{\mu} \otimes v_{-\mu}\right)\right)\left(v_{\mu} \otimes v_{-\mu}\right)=b$. Finally apply the fact that $v_{\mu} \otimes v_{-\mu}$ generates $V(\mu) \otimes V(-\mu)$ as a $U$ module.

3.7. Let $S(\nu, V)$ denote the $V$ isotypical component of the socle of $\operatorname{End} V(\nu)$. Through the embeddings $Y_{\nu, \mu}$, we may regard the $S(\nu, V)$, for $\nu \in P_{0}^{+}(\pi)$, as forming a lattice of subspaces (of some common limiting space obtained by taking $\nu$ sufficiently large). As noted in [JT, proof of Proposition 3.3], [J2, Corollary 7.3.3] extends for $V$ admissible to show that this lattice is distributive. (Finite dimensionality of weight spaces is needed to get the downward induction started.) Thus, given $\mu, \nu_{1}, \ldots, \nu_{n}$ in $P_{0}^{+}(\pi)$,

$$
S(\mu, V) \cap\left(\sum_{j=1}^{n} S\left(\nu_{j}, V\right)=\sum_{j=1}^{n} S\left(\mu \cap \nu_{j}, V\right) .\right.
$$

Lemma. The filtration $\mathcal{F}$ is distributive on $V_{0}$. 
Proof. Suppose that

$$
v \in \mathcal{F}^{\nu}\left(V_{0}\right) \cap \sum_{\gamma} \mathcal{F}^{\gamma}\left(V_{0}\right)
$$

Without loss of generality, we can assume there is a weight $\mu$ such that $\nu \prec \mu$ and each $\gamma$ also satisfies $\gamma \prec \mu$. Furthermore, we can choose $\mu$ large enough so that $\mathcal{F}^{\mu}\left(V_{0}\right)=V_{0}$. Since $v \in \mathcal{F}^{\nu}\left(V_{0}\right)$, we obtain

$$
\begin{aligned}
\varphi_{v}^{\mu, *}\left(V^{\#}\right) & =\left(\Phi_{\mu}\left(i_{\mu, \nu}(v)\right)\right)\left(V^{\#}\right)=\left(\Psi_{\mu, \nu}^{*}\left(\Phi_{\nu}(v)\right)\right)\left(V^{\#}\right) \\
& =\psi_{\mu, \nu}^{*}\left(\varphi_{v}^{\nu, *}\left(V^{\#}\right)\right) \subset \psi_{\mu, \nu}^{*}(S(\nu, V),
\end{aligned}
$$

by Lemma 3.4. Consequently

$$
\varphi_{v}^{\mu, *}\left(V^{\#}\right) \subseteq \psi_{\mu, \nu}^{*}(S(\nu, V)) \cap \sum_{\gamma} \psi_{\mu, \gamma}^{*}(S(\gamma, V))=\sum_{\gamma} \psi_{\mu, \mu \cap \gamma}^{*}(S(\nu \cap \gamma, V))
$$

by $(*)$. Hence $\varphi_{v}^{\mu, *}=\sum_{\gamma} \varphi_{w_{\gamma}}^{\mu, *}$ for suitable choices of $w_{\gamma} \in \mathcal{F}^{\nu \cap \gamma}\left(V_{0}\right)$. Since $\Phi_{\mu}$ is linear, it follows that $\varphi_{v}^{\mu, *}=\varphi_{w}^{\mu, *}$ where $w=\sum_{\gamma} w_{\gamma}$. But the map $\Phi_{\mu}$ is an isomorphism so $v=w$. This gives one required inclusion. The opposite one is trivial.

\section{The Factorization Theorem}

4.1. Let $V$ be a simple integrable admissible $U$ module. For each $\beta \in \mathbb{Z} \pi$, let $V_{\beta}$ denote the $\beta$ weight space of $V$. Then $\operatorname{dim} V_{\beta}$ is finite and in particular the formal character

$$
\operatorname{ch} V=\sum_{\beta \in \mathbb{Z}_{\pi}}\left(\operatorname{dim} V_{\beta}\right) e^{\beta}
$$

is defined. Since $V$ is integrable, ch $V$ is $W$ invariant. Furthermore since $\operatorname{dim} V_{0}$ is finite, the integrability of $V$ implies that for each $\alpha \in \pi$ one has $\operatorname{dim} V_{n \alpha}=0$ for all $n \in \mathbb{N}$ sufficiently large. Since $\Delta_{r e}=W \pi$ by definition, it follows that there exists an integer $s>0$ such that $\operatorname{dim} V_{t \beta}=0$, for all $t \geq s$ and for all $\beta \in \Delta_{r e}^{+}$.

Set

$$
\lambda_{V}:=\sum_{\mu \in P_{0}^{+}(\pi)} \mu \operatorname{dim} \mathcal{F}_{\mu}\left(V_{0}\right) .
$$

In [JT, 3.6], it is shown that $\mathcal{P}_{V}$ is an element of $\tau\left(-2 \lambda_{V}\right) A_{\pi}$ with highest degree term a nonzero scalar multiple of $\tau\left(-2 \lambda_{V}\right)$ with $\mathbb{H}_{V}$ chosen as in [JT, 3.6]. We use this definition in Section 4.3 to obtain a formula for $\alpha^{\vee}\left(-2 \lambda_{V}\right), \alpha \in \pi$, which closely resembles the semisimple case $([\mathrm{J} 3,8.2 .10(*)])$.

4.2. Fix $\alpha \in \pi$ and let $\omega_{\alpha}$ be the choice of fundamental weight corresponding to $\alpha$. Let $s$ be a positive integer and let $V_{0}^{s}$ be the subspace of $V_{0}$ annihilated by $e_{\alpha}^{s+1}$. Given $\nu \in P_{0}^{+}(\pi)$, set $\nu_{s}=\nu \cap s \omega_{\alpha}$. Note that $\mathcal{F}^{\nu}\left(V_{o}^{s}\right)=\mathcal{F}^{\nu_{s}}\left(V_{0}\right)$ for all $\nu \in P_{0}^{+}(\pi)$. Thus Lemma 3.7 implies that the filtration $\mathcal{F}$ is distributive on $V_{0}^{s}$.

Lemma. $\lambda_{V}=\sum_{\mu} \sum_{\left\{s \mid \alpha^{\vee}(\mu)=s\right\}} \mu \operatorname{dim} \mathcal{F}_{\mu}\left(V_{0}^{s}\right)$. 
Proof. Fix $\mu$ and set $s=\alpha^{\vee}(\mu)$. Consider $\nu \in P_{0}^{+}(\pi)$ such that $\nu \preccurlyeq \mu$. Note that $\nu=\nu_{s}$ and so $\mathcal{F}^{\nu}\left(V_{0}\right)=\mathcal{F}^{\nu}\left(V_{0}^{s}\right)$. It follows that $\mathcal{F}_{\mu}\left(V_{0}\right)=\mathcal{F}_{\mu}\left(V_{0}^{s}\right)$.

4.3. Consider the $T$ submodule

$$
V^{(\alpha)}:=\bigoplus_{n \in \mathbb{Z}} V_{n \alpha}
$$

of $V$. Clearly $V^{(\alpha)}$ is a $U_{\alpha}$ submodule of $V$ and integrable, since $V$ itself is integrable. Hence $V^{(\alpha)}$ is a direct sum

$$
V^{(\alpha)}=\bigoplus_{i} V^{(\alpha), i}
$$

of simple $U_{\alpha}$ submodules of odd dimension, say $\operatorname{dim} V^{(\alpha), i}=2 n_{i}+1$. Moreover $V^{(\alpha), i}$ has one dimensional weight spaces of weights $\pm t \alpha: 0 \leq t \leq n_{i}$. In particular the $V_{0}^{(\alpha), i}$ are one dimensional and their direct sum is $V_{0}$.

Set $V_{0, s}=\sum_{n_{j}=s} V_{0}^{(\alpha), j}$. Note that $\sum_{n=1}^{\infty} \operatorname{dim} V_{n \alpha}=\sum_{s=1}^{\infty} s \operatorname{dim} V_{0, s}$. Furthermore, $\operatorname{dim} V_{0}^{s}-\operatorname{dim} V_{0}^{s-1}$ is equal to $\operatorname{dim} V_{0, s}$ since $V_{0, s}$ is a vector space complement to $V_{0}^{s-1}$ in $V_{0}^{s}$.

Lemma. For all $\alpha \in \pi$ one has $\alpha^{\vee}\left(\lambda_{V}\right)=\sum_{n=1}^{\infty} \operatorname{dim} V_{n \alpha}$.

Proof. By Lemma 3.3, $\operatorname{dim} \operatorname{gr}_{\mathcal{F}}\left(V_{0}^{s}\right)=\operatorname{dim} V_{0}^{s}$. Thus, Lemma 4.2 implies that

$$
\alpha^{\vee}\left(\lambda_{V}\right)=\sum_{s} s \sum_{\left\{\mu \mid \alpha^{\vee}(\mu)=s\right\}} \operatorname{dim} \mathcal{F}_{\mu}\left(V_{0}^{s}\right) .
$$

Note that

$$
\begin{aligned}
& \sum_{\mu} \mathcal{F}_{\mu}\left(V_{0}^{s}\right)=\sum_{\left\{\mu \mid \alpha^{\vee}(\mu)=s\right\}} \mathcal{F}_{\mu}\left(V_{0}^{s}\right)+\sum_{\left\{\mu \mid \alpha^{\vee}(\mu)<s\right\}} \mathcal{F}_{\mu}\left(V_{0}^{s}\right) \\
& =\sum_{\left\{\mu \mid \alpha^{\vee}(\mu)=s\right\}} \mathcal{F}_{\mu}\left(V_{0}^{s}\right)+\sum_{\mu} \mathcal{F}_{\mu}\left(V_{0}^{s-1}\right) .
\end{aligned}
$$

Since these are all direct sums,

$$
\operatorname{dim} V_{0}^{s}=\sum_{\left\{\mu \mid \alpha^{\vee}(\mu)=s\right\}} \operatorname{dim} \mathcal{F}_{\mu}\left(V_{0}^{s}\right)+\operatorname{dim} V_{0}^{s-1} .
$$

Thus $\alpha^{\vee}\left(\lambda_{V}\right)=\sum_{s} s\left(\operatorname{dim} V_{0}^{s}-\operatorname{dim} V_{0}^{s-1}\right)$, so substitution from the paragraph preceding the lemma gives the required assertion.

4.4. Recall the notation of Section 2.6. Fix $v_{i} \in V_{0}^{(\mu), i}$ nonzero. Recall that any choice of the harmonic space $\mathbb{H}_{V}$ is a direct sum of $\operatorname{dim} V_{0}$ copies of $V$. The set $\left\{\varphi_{j}\right\}$ is a basis for $\operatorname{Hom}\left(V, \mathbb{H}_{V}\right)$ and $v_{i, j}=\varphi_{j}\left(v_{i}\right)$. Fix $\alpha \in \pi$ and recall the construction of 4.3 .

Lemma. For all $i, j=1,2, \cdots, \operatorname{dim} V_{0}$ one has $\mathcal{P}\left(v_{i, j}\right)=S\left(n_{i}\right) m_{i, j}$ for some $m_{i, j} \in U^{\alpha}$. 
Proof. Clearly $V^{\prime}:=\mathcal{P}_{\alpha}\left(\varphi_{j}\left(V^{(\alpha), i}\right)\right)$ is either zero or a simple ad $U_{\alpha}$ submodule of $R\left(\hat{U}_{\alpha}\right)$ of dimension $2 n_{i}+1$. Hence $\mathcal{P}\left(\varphi_{j}\left(V^{(\alpha), i}\right)\right)=\mathcal{P}\left(V^{\prime}\right) \subset S\left(n_{i}\right) U^{\alpha}$, by 2.7 .

4.5. Comparing $(*)$ and $(* *)$ of 4.3 , we have

$$
Q_{\alpha}:=\prod_{i=1}^{\operatorname{dim} V_{0}} S\left(n_{i}\right)=\prod_{n=1}^{\infty}\left(r_{\alpha}-q^{n(\alpha, \alpha)} r_{\alpha}^{-1}\right)^{\operatorname{dim} V_{n \alpha}} .
$$

Corollary. For each $\alpha \in \pi$ one has $\mathcal{P}_{V}=Q_{\alpha} Q^{\alpha}$ for some $Q^{\alpha} \in U^{\alpha}$.

Proof. Indeed by definition

$$
\mathcal{P}_{V}=\operatorname{det} \mathcal{P}\left(v_{i, j}\right)=\left(\prod_{i=1}^{\operatorname{dim} V_{0}} S\left(n_{i}\right)\right) \operatorname{det} m_{i, j}
$$

through 4.4. Since $U^{\alpha}$ is a subalgebra of $\hat{U}^{0}$ the conclusion obtains.

4.6. Set

$$
Q_{V}=\tau\left(-2 \lambda_{V}\right) \prod_{\beta \in \Delta_{r e}^{+}} \prod_{n=1}^{\infty}\left(1-q^{-n(\beta, \beta)} r_{\beta}^{2}\right)^{\operatorname{dim} V_{n \beta}}
$$

Lemma. For all $\alpha \in \pi$ one has $Q_{V} Q_{\alpha}^{-1} \in U^{\alpha}$.

Proof. Set $\Delta_{\alpha}^{+}:=\Delta_{r e}^{+} \backslash\{\alpha\}$. It is $s_{\alpha}$ stable. From the definitions of $Q_{V}$ and $Q_{\alpha}$ we have

$$
Q_{V} Q_{\alpha}^{-1}=\tau\left(-2 \lambda_{V}+\sum_{n=1}^{\infty} \alpha \operatorname{dim} V_{n \alpha}\right) \prod_{\beta \in \Delta_{\alpha}^{+}} \prod_{n=1}^{\infty}\left(1-q^{-n(\beta, \beta)} r_{\beta}^{2}\right)^{\operatorname{dim} V_{n \beta}},
$$

up to a power of $q$. By 4.3, the first factor lies in $T^{\alpha}$. Since $s_{\alpha} \cdot r_{\beta}=r_{s_{\alpha} \beta}$ and $\operatorname{dim} V_{n \beta}=\operatorname{dim} V_{n s_{\alpha} \beta}$, the second factor is $s_{\alpha}$. invariant and lies in $\hat{U}^{0}$.

4.7. Recall [JT, 3.6] (see Section 4.1) that $\mathcal{P}_{V} \in \tau\left(-2 \lambda_{V}\right) A_{\pi}$, for a choice of $\mathbb{H}_{V}$ described there. For an arbitrary choice $\mathcal{P}_{V}$ may be modified by a non-zero element of $K$. However such an element is $W$. invariant and so may be ignored.

Theorem. One has $\mathcal{P}_{V}=Q_{V}$, up to a non-zero element of $K$.

Proof. That $\mathcal{P}_{V} \neq 0$ is just [JT, Proposition 3.6] which shows in the language of Section 4.1 that $\tau\left(-2 \lambda_{V}\right)$ is the highest weight term of $\mathcal{P}_{V}$ up to a nonzero scalar. It is clear that $\mathcal{P}_{V} Q_{V}^{-1} \in A_{\pi}$. Hence by 2.3 it suffices to show that this expression is $W$. invariant. It is enough for the expression to be $s_{\alpha}$. invariant for each $\alpha \in \pi$. Yet by 4.5 and 4.6 one has $\mathcal{P}_{V} Q_{\alpha}^{-1}, Q_{V} Q_{\alpha}^{-1} \in U^{\alpha}$. Hence the assertion. 


\section{The Multiplicity Free Case}

5.1. Recall that for the choice of $\mathbb{H}_{V}$ made in [JT, 3.6] the entries $\mathcal{P}\left(v_{i, j}\right)$ of the PRV determinant lie in $A_{\pi}$, up to multiples by elements in $T$. If we make a more general choice, then in a given column of the matrix $\mathcal{P}\left(v_{i, j}\right)$ the entries may be multiplied by a fixed non-zero element of $K$. Indeed this depends on the choice of the homomorphism $\varphi_{j}: V \rightarrow R(\hat{U})_{V}$. The linear span over $K$ of their images defines $\mathbb{H}_{V}$. However since $K A_{\pi}=T A_{\pi}$, the first assertion is still valid. The choice of these scalars in $K$ in the definition of $\varphi_{j}$ will be seen to be a delicate point.

5.2. For all $\beta \in \mathbb{N} \pi$, let $|\beta|$ denote the sum of its coefficients (with respect to $\pi)$. Given $c_{\beta} \in k((q)): \beta \in \mathbb{N} \pi$, we say that the sum $\sum_{\beta \in \mathbb{N}_{\pi}} c_{\beta}$ converges (in the Krull topology) if for each $n \in \mathbb{N}$ there exists $m \in \mathbb{N}$ such that $c_{\beta} \in q^{n} k[[q]]$, for all $\beta \in \mathbb{N} \pi$ satisfying $|\beta| \geq m$.

Let $a \in A_{\pi}$. We may write $a$ as an infinite sum

$$
a=\sum_{\beta \in \mathbb{N}_{\pi}} c_{\beta} t_{\beta}^{2}: c_{\beta} \in k(q) .
$$

We say that $a$ is defined at $\nu \in P(\pi)$, if $\sum_{\beta \in \mathbb{N} \pi} c_{\beta} q^{2(\beta, \nu)}$ converges in the Krull topology to an element of $k((q))$ which we denote by $a(\nu)$. It is clear that if $a$ is defined at 0 , then it is defined at all $\nu \in P^{+}(\pi)$.

A basic question is whether the $\mathcal{P}\left(v_{i, j}\right)$ are defined at 0 . For this we must obviously make a correct choice of scalars in the definition of the homomorphisms $\varphi_{j}: V \rightarrow R(\hat{U})_{V}$, equivalently in the choice of the harmonic space $\mathbb{H}_{V}$ identified as the $K$ linear span of the $\varphi_{j}(V)$. The most obvious choice (made in [JT, 3.6]) may not be appropriate.

Following $[\mathrm{J} 3,6.1]$ let $\mathcal{O}_{\mathbb{P}}$ denote the full subcategory of $\mathcal{O}$ whose simple factors have highest weights in $W \cdot P^{+}(\pi)$. A straightforward modification of [J3, 6.13] shows that if the $\mathcal{P}\left(v_{i, j}\right)$ are defined at 0 , then the corresponding harmonic space $\mathbb{H}_{V}$ is defined on all $M \in O b \mathcal{O}_{\mathbb{P}}$. This means (but see [J3, 6.2] for more details) that we may extend the action of $U$ on $M$ to elements of $\mathbb{H}_{V}$, where we replace an infinite sum of torus elements on a weight vector of weight $\nu$, by its value at $\nu$.

5.3. Recall the definition of $Q_{V}$ from Section 4.6.

Lemma. $Q_{V}$ is defined at 0 .

Proof. By 4.1, there exists a positive integer $s$ such that up to a multiple of $T$, we can write

$$
Q_{V}=\prod_{\beta \in \Delta_{r e}^{+}} \prod_{n=1}^{s}\left(1-q^{2(\rho, \beta)-n(\beta, \beta)} t_{\beta}^{2}\right)^{\operatorname{dim} V_{n \beta}} .
$$

Except for finitely many positive real roots one has $2(\rho, \beta)>s(\beta, \beta)$. The required assertion follows. 
5.4. Combining 4.7 and 5.3, it follows that up to a choice of scalars (equivalently up to a choice of $\left.\mathbb{H}_{V}\right) \mathcal{P}_{V}$ is defined at 0 . However there will obviously be many bad choices for which $\mathbb{H}_{V}$ will not be defined on some $M \in O b \mathcal{O}_{\mathbb{P}}$. Conjecturally there is always a choice so that this does hold; but the question seems to be rather delicate. Yet if $\mathbb{H}_{V}$ is multiplicity-free (equivalently if $\operatorname{dim} V_{0}=1$ ) then of course we can choose the scalar to obtain this desired conclusion.

5.5. Take $\lambda \in P^{+}(\pi)$ and recall that $V(\lambda)$ is the simple highest weight module of highest weight $\lambda$. Take $a \in \operatorname{End} V(\lambda)$ of weight 0 . Recall [J3,6.5]. The quantum trace $\operatorname{tr}_{q}(a, V(\lambda))$ of $a$ on $V(\lambda)$ is the infinite sum

$$
\sum_{\mu \in \mathbb{N}_{\pi}} \operatorname{tr}\left(a \mid V(\lambda)_{\lambda-\mu}\right) q^{-2(\rho, \lambda-\mu)}
$$

given that the latter converges in the Krull topology. In this case we say that $\operatorname{tr}_{q}(a, V(\lambda))$ converges (in the Krull topology). This convergence condition is equivalent to the assertion that

$$
c_{\lambda}(a):=\sum_{\mu \in \mathbb{N}_{\pi}} \operatorname{tr}\left(a \mid V(\lambda)_{\lambda-\mu}\right) q^{-2(\rho, \lambda-\mu)} \tau(-2(\lambda-\mu))
$$

is defined at 0 . We say that $\operatorname{tr}_{q}(a, V(\lambda))$ converges (in the Krull topology) up to $K$, if there exists $0 \neq b \in K$ such that $b c_{\lambda}(a)$ is defined at 0 . We can express the conclusion of 5.4 in the following manner.

Let $V$ be a simple admissible integral module with a one dimensional zero weight space. By $[\mathrm{J} 3,5.12]$ one has $\operatorname{dim} \operatorname{Hom}_{\mathfrak{g}}(V, \operatorname{End} V(\lambda)) \leq 1$, for all $\lambda \in$ $P^{+}(\pi)$. Moreover there is a unique minimal $\mu \in P_{0}^{+}(\pi)$ s.t. $\operatorname{Hom}_{\mathfrak{g}}(V, \operatorname{End} V(\lambda))=$ 1 , for all $\lambda \succcurlyeq \mu$ and zero otherwise. In the former case we can view $a \in V_{0} \backslash\{0\}$ as an element of $\operatorname{End} V(\lambda)_{\lambda-\mu}$, for all $\mu \in \mathbb{N} \pi$.

Corollary. Retain the above hypotheses and notation. Then $\operatorname{tr}_{q}(a, V(\lambda)): \lambda \succcurlyeq \mu$ converges up to $K$.

Proof. Recall (2.6) that $R(\hat{U})_{V}$ is free over $Z(R(\hat{U}))$ and [J3, 6.2] that the latter is spanned over $K$ by the elements $z_{\mu}: \nu \in P^{+}(\pi)$. The latter acts by $\operatorname{tr}_{q}(1, V(\nu)) \in k((q))$ on $V(0)$. Consequently, it is enough to take $\lambda=\mu$ and then the assertion is just the observation made in 5.4.

5.5. Retain the hypotheses and notation of 5.5. Our preliminary investigation $[\mathrm{J} 5,3.2]$ indicated that the quantum trace $\operatorname{tr}_{q}(a, V(\mu))$ will not in general converge. In any case we calculated the $q=1$ limit of the PRV determinant in the simplest possible case (basically the adjoint representation for $\widehat{\mathfrak{s l}}_{2}$ ) by the use of such traces and obtained an expression which agreed with the $q=1$ limit of $Q_{V}$ up to factors involving $\tau(\delta)^{2}$. This is of course no contradiction; it indicates that the choice of $\mathbb{H}_{V}$ by the procedure of $[\mathrm{JT}, 3.6]$ may not be the best one. A similar calculation for a second $\widehat{\mathfrak{s l}}_{2}$ module with a one dimensional zero weight space is given in [GJ]. The conclusion is similar. These special cases are almost 
as difficult than our general theorem. However they do give a little extra infor-

mation on imaginary root factors and indicate that the choice of scalars (or the harmonic space) is a delicate point.

\section{References}

[CP] V. Chari and A. Pressley, Integrable representations of twisted affine Lie algebras, J. Algebra 113 (1988), no. 2, 1-18.

[G] J. Greenstein, Characters of bounded $\hat{\mathfrak{s l}}(2)$ modules, J. Algebra 230 (2000), no. 2, 540557.

[GJ] J. Greenstein and A. Joseph, A Chevalley-Kostant presentation of basic modules and the associated affine KPRV determinants at $q=1$, Bull. Sci. Math. 125 (2001), no. 2, 85-108.

[J1] A. Joseph, Enveloping algebras: problems old and new, Lie theory and geometry, 385413, Progr. Math., 123, Birkhäuser Boston, Boston, MA, 1994.

[J2] _ Quantum groups and their primitive ideals, Ergebnisse der Mathematik und ihrer Grenzgebiete (3), 29. Springer-Verlag, Berlin, 1995.

[J3] — A completion of the quantized enveloping algebra of a Kac-Moody algebra, J. Algebra 214 (1999), no. 1, 237-275.

[J4] The admissibility of simple bounded modules for an affine Lie algebra, Algebr. Represent. Theory 3 (2000), no. 2, 131-149.

[J5] $\longrightarrow$ On an affine quantum KPRV determinant at $q=1$, Bull. Sci. Math. 125 (2001), no. 1, 1-26.

[JL] A. Joseph and G. Letzter, Verma module annihilators for quantized enveloping algebras, Ann. Sci. École Norm. Sup. (4) 28 (1995), no. 4, 493-526.

[JT] A. Joseph and D. Todoric, On the quantum KPRV determinants for semisimple and affine Lie algebras, to appear in Algebr. Represent. Theory.

[K] V.G. Kac, Infinite-dimensional Lie algebras. Second edition, Cambridge University Press, Cambridge, MA, 1985.

[Ke] M. S. Kébé, O-algèbres quantiques, C. R. Acad. Sci. Paris, Sér. I Math. 322 (1996), no. 1, 1-4.

[PRV] K. R. Parthasarathy, R. Ranga Rao, and V.S. Varadarajan, Representations of complex semisimple Lie groups and Lie algebras, Ann. of Math. (2) 85 (1967), 383-429.

The Weizmann Institute of Science, Rehovot 76100, Israel.

Institut de Mathématiques, UMR 7586, 175 Rue du Chevaleret, Plateau 7D, 75013

PARIs Cedex, France.

E-mail address: joseph@ccr.jussieu.fr

Department of Mathematics, Virginia Polytechnic Institute and State UniverSity, Blacksburg, Virginia 24061-0123, U.S.A.

E-mail address: letzter@calvin.math.vt.edu 